\title{
Virtual Correlations in Single Qutrit
}

\author{
Alexey A. Strakhov ${ }^{1}$ and Vladimir I. Man'ko ${ }^{1,2}$ \\ ${ }^{1}$ Moscow Institute of Physics and Technology, Institutskii per. 9, Dolgoprudny, Moscow Oblast 141700, Russia \\ ${ }^{2}$ P.N. Lebedev Physical Institute, Russian Academy of Sciences, Leninskii Prospect 53, Moscow 119991, Russia
}

Correspondence should be addressed to Vladimir I. Man’ko; manko@sci.lebedev.ru

Received 19 June 2016; Revised 14 September 2016; Accepted 21 September 2016

Academic Editor: Giorgio Kaniadakis

Copyright (c) 2016 A. A. Strakhov and V. I. Man'ko. This is an open access article distributed under the Creative Commons Attribution License, which permits unrestricted use, distribution, and reproduction in any medium, provided the original work is properly cited.

We construct the positive invertible map of the mixed states of a single qutrit onto the antisymmetrized bipartite qutrit states (quasifermions). It is shown that using this one-to-one correspondence between qutrit states and states of two three-dimensional quasifermions one may attribute hidden entanglement to a single mixed state of qutrit.

\section{Introduction}

Quantum entanglement [1] is one of the most valuable nonclassical resources in quantum information science. By virtue of entanglement the protocols of superdense coding [2] and quantum teleportation [3] were discovered. Entangled states are essential in quantum computing $[4,5]$ and are used in various cryptography protocols $[6,7]$. Entangled states are also used in more applied areas of physics such as quantum metrology $[8,9]$.

Naturally it is hard to prepare and maintain maximally entangled states. In order to control the amount of entanglement in quantum systems several measures were proposed. In case of bipartite pure states an entropy of entanglement [10] was proved to be an asymptotic measurement of distillating maximally entangled states by means of local operations and classical communication (LOCC) from the supply of initial pure states and vice versa. The case of mixed states is more complicated. In particular for mixed states the following measures were introduced: the asymptotic entanglement of formation and distillation [11] and the relative entropy of entanglement [12]. However, all of them include variational step and thus are generally hardly calculable (notable exceptions are mixed qubit states fully characterized by concurrence [13]). From the Peres-Horodecki criterion [14, 15] the notions of negativity and logarithmical negativity [16-18] emerged. They are relatively easy calculable and the latter provides an upper bound of distillable entanglement. Negativity will be used further in the paper.

Recently several attempts to study quantum correlations of noncomposite qudit systems were conducted [19-23]. The physical realization of such systems is convenient with nonlinear quantum circuits on Josephson junctions [24, 25]. This paper is an attempt to study correlations between different degrees of freedom in qutrit via the invertible map to a bipartite system of antisymmetrized states of qutrits (as the dimension of them is odd we shall call them quasifermions). The source of correlations in such case would be antisymmetrization, that is, restriction to the subspace of the whole tensor product space of two qutrits. Actually using the same method one can construct an isomorphism between sixdimensional noncomposite qudit and two indistinguishable four-dimensional fermions. Correlations between particles and modes in the systems of indistinguishable particles were recently studied [26-30]. For mixed states of indistinguishable four-dimensional fermions obtained by the isomorphism discussed in the present paper both the dualization concurrence and Slater number will show that the states are separable in the sense of antisymmetrized fermionic subspace. However, we would like to emphasize that due to the virtuality of the division of the noncomposite system into bipartite quasifermion system it is not necessary to treat these quasifermions as indistinguishable particles. One might view correlations resulting from antisymmetrization as internal 
correlations between different degrees of freedom of the initial qudit. Thus one might treat the initial six-dimensional mixed state as a fully separable state of indistinguishable Dim $=4$ fermions or as an entangled state of distinguishable Dim $=4$ particles. The present paper will be about analyzing negativity of qutrit viewed as an antisymmetrized biqutrit state. An open question in such approach is operating with the corresponding virtual subsystems.

\section{Partial Trace in Matrix Representation}

The state of a subsystem of a quantum system is defined by an operation called partial trace, taking mean value over states of another subsystem:

$$
\begin{aligned}
\rho_{\mathscr{I}} & =\operatorname{Tr}_{\mathscr{F} \backslash \mathscr{I}}\left[\rho_{\mathscr{F}}\right] \Longleftrightarrow \\
\left\langle i_{1}\left|\rho_{\mathscr{I}}\right| i_{2}\right\rangle & =\sum_{|j\rangle \in \mathscr{F} \backslash \mathscr{I}}\left\langle i_{1}\left|\left\langle j\left|\rho_{\mathscr{F}}\right| j\right\rangle\right| i_{2}\right\rangle,
\end{aligned}
$$

where $\left|i_{1}\right\rangle$ and $\left|i_{2}\right\rangle$ are basis vectors of the subsystem $\mathscr{I}$ and $|j\rangle$ is basis vectors of the subsystem $\mathscr{J} \backslash \mathscr{I}$. Despite the explicit choice of basis vectors of space $\mathscr{J}$, partial trace is invariant with respect to unitary transformations of $\mathscr{I}$.

Let us consider a space $\mathscr{J}=\mathrm{H}_{3} \otimes \mathrm{H}_{2}$ and choose a lexicographically ordered basis $\left|e_{1}\right\rangle=\left|a_{1}\right\rangle \otimes\left|b_{1}\right\rangle,\left|e_{2}\right\rangle=$ $\left|a_{1}\right\rangle \otimes\left|b_{2}\right\rangle, \ldots,\left|e_{6}\right\rangle=\left|a_{3}\right\rangle \otimes\left|b_{2}\right\rangle$. Then an arbitrary state $\rho$ will be presented as matrix:

$$
\rho=\left(\begin{array}{cccccc}
\rho_{11} & \rho_{12} & \rho_{13} & \rho_{14} & \rho_{15} & \rho_{16} \\
\rho_{21} & \rho_{22} & \rho_{23} & \rho_{24} & \rho_{25} & \rho_{26} \\
\rho_{31} & \rho_{32} & \rho_{33} & \rho_{34} & \rho_{35} & \rho_{36} \\
\rho_{41} & \rho_{42} & \rho_{43} & \rho_{44} & \rho_{45} & \rho_{46} \\
\rho_{51} & \rho_{52} & \rho_{53} & \rho_{54} & \rho_{55} & \rho_{56} \\
\rho_{61} & \rho_{62} & \rho_{63} & \rho_{64} & \rho_{65} & \rho_{66}
\end{array}\right) .
$$

Formula (1) then might be represented by square block matrices whose sizes are determined by the numbers of dimensions of spaces $\mathrm{H}_{3}$ and $\mathrm{H}_{2}$ :

$$
\begin{aligned}
\rho & =\left(\begin{array}{ll|ll|ll}
\rho_{11} & \rho_{12} & \rho_{13} & \rho_{14} & \rho_{15} & \rho_{16} \\
\rho_{21} & \rho_{22} & \rho_{23} & \rho_{24} & \rho_{25} & \rho_{26} \\
\hline \rho_{31} & \rho_{32} & \rho_{33} & \rho_{34} & \rho_{35} & \rho_{36} \\
\rho_{41} & \rho_{42} & \rho_{43} & \rho_{44} & \rho_{45} & \rho_{46} \\
\hline \rho_{51} & \rho_{52} & \rho_{53} & \rho_{54} & \rho_{55} & \rho_{56} \\
\rho_{61} & \rho_{62} & \rho_{63} & \rho_{64} & \rho_{65} & \rho_{66}
\end{array}\right) \\
& =\left(\begin{array}{lll}
R_{11} & R_{12} & R_{13} \\
R_{21} & R_{22} & R_{23} \\
R_{31} & R_{32} & R_{33}
\end{array}\right) ;
\end{aligned}
$$

$$
\begin{aligned}
& \rho_{1}=\left(\begin{array}{ccc}
\operatorname{Tr} R_{11} & \operatorname{Tr} R_{12} & \operatorname{Tr} R_{13} \\
\operatorname{Tr} R_{21} & \operatorname{Tr} R_{22} & \operatorname{Tr} R_{23} \\
\operatorname{Tr} R_{31} & \operatorname{Tr} R_{32} & \operatorname{Tr} R_{33}
\end{array}\right), \\
& \rho_{2}=R_{11}+R_{22}+R_{33},
\end{aligned}
$$

where $\rho_{1}$ is a state of the first subsystem $H_{3}$ and $\rho_{2}$ is a state of the second subsystem $\mathrm{H}_{2}$. Partial traces in matrix form will be constantly used further in this paper.

\section{Qutrit as $\mathscr{H}=H_{3} \wedge H_{3}$ Quasifermionic System}

Let us pick a basis $\left\{\left|e_{1}\right\rangle,\left|e_{2}\right\rangle,\left|e_{3}\right\rangle\right\}$ in an arbitrary threedimensional state space $Q$. The general mixed state of qutrit is described by a density operator $\rho_{I} \in S(\mathbb{Q}): \rho_{I}=$ $\sum_{i, j=1}^{3}\left(\rho_{I}\right)_{i j}\left|e_{i}\right\rangle\left\langle e_{j}\right|$, in matrix form:

$$
\rho_{I}=\left(\begin{array}{lll}
\rho_{11} & \rho_{12} & \rho_{13} \\
\rho_{21} & \rho_{22} & \rho_{23} \\
\rho_{31} & \rho_{32} & \rho_{33}
\end{array}\right) .
$$

Now we will consider a system of two three-dimensional quasifermions, antisymmetrized qutrits. Mathematically it is an exterior product, that is, the quotient of the tensor product by the subspace generated by $x \otimes x$ elements, of two threedimensional spaces: $\mathscr{H}=H_{3} \wedge H_{3}$. Let us pick orthonormal basis vectors in $H_{3}$ as $|1\rangle,|2\rangle,|3\rangle$. Then in the normalized basis $\left\{\left|g_{1}\right\rangle,\left|g_{2}\right\rangle,\left|g_{3}\right\rangle\right\}$ of the exterior product an arbitrary pure state $|\Psi\rangle \in \mathscr{H}$ looks as follows:

$$
\begin{aligned}
& |\Psi\rangle=a_{1}\left|g_{1}\right\rangle+a_{2}\left|g_{2}\right\rangle+a_{3}\left|g_{3}\right\rangle, \\
& \left|g_{1}\right\rangle=\frac{1}{\sqrt{2}}|1\rangle \wedge|2\rangle=\frac{1}{\sqrt{2}}(|1\rangle \otimes|2\rangle-|2\rangle \otimes|1\rangle), \\
& \left|g_{2}\right\rangle=\frac{1}{\sqrt{2}}|1\rangle \wedge|3\rangle=\frac{1}{\sqrt{2}}(|1\rangle \otimes|3\rangle-|3\rangle \otimes|1\rangle), \\
& \left|g_{3}\right\rangle=\frac{1}{\sqrt{2}}|2\rangle \wedge|3\rangle=\frac{1}{\sqrt{2}}(|2\rangle \otimes|3\rangle-|3\rangle \otimes|2\rangle) .
\end{aligned}
$$

We will further omit the $\otimes$ symbol of tensor product. Thus we see that the space $\mathscr{H}$ is isomorphic to qutrit space Q. The general form of density operator $\rho_{O} \in S(\mathscr{H})$ on space $\mathscr{H}$ is $\rho_{O}=\sum_{i, j=1}^{3}\left(\rho_{O}\right)_{i j}\left|g_{i}\right\rangle\left\langle g_{j}\right|$, so we can introduce an isomorphism between sets of mixed states $S(\mathscr{Q})$ and $S(\mathscr{H})$ by equalizing matrix elements $\left(\rho_{I}\right)_{i j}=\left(\rho_{O}\right)_{i j}=\rho_{i j}$ for all $i, j=1,2,3$. 
As we already mentioned, $\rho_{\mathrm{O}}$ is a state in antisymmetrized subspace of the tensor product space $\mathrm{H}_{3} \otimes H_{3}$. Let us observe this state in context of the whole space $\mathrm{H}_{3} \otimes H_{3}$. It is easy to verify that its matrix representation is as follows:

$$
\rho_{O}^{\prime}=\frac{1}{2}
$$

$$
\cdot\left(\begin{array}{ccccccccc}
0 & 0 & 0 & 0 & 0 & 0 & 0 & 0 & 0 \\
0 & \rho_{11} & \rho_{12} & -\rho_{11} & 0 & \rho_{13} & -\rho_{12} & -\rho_{13} & 0 \\
0 & \rho_{21} & \rho_{22} & -\rho_{21} & 0 & \rho_{23} & -\rho_{22} & -\rho_{23} & 0 \\
0 & -\rho_{11} & -\rho_{12} & \rho_{11} & 0 & -\rho_{13} & \rho_{12} & \rho_{13} & 0 \\
0 & 0 & 0 & 0 & 0 & 0 & 0 & 0 & 0 \\
0 & \rho_{31} & \rho_{32} & -\rho_{31} & 0 & \rho_{33} & -\rho_{32} & -\rho_{33} & 0 \\
0 & -\rho_{21} & -\rho_{22} & \rho_{21} & 0 & -\rho_{23} & \rho_{22} & \rho_{23} & 0 \\
0 & -\rho_{31} & -\rho_{32} & \rho_{31} & 0 & -\rho_{33} & \rho_{32} & \rho_{33} & 0 \\
0 & 0 & 0 & 0 & 0 & 0 & 0 & 0 & 0
\end{array}\right) .
$$

Now using the partial trace operation, introduced in the previous section, we are able to obtain single three-dimensional quasifermionic states:

$$
\rho_{1}=\rho_{2}=\frac{1}{2}\left(\begin{array}{ccc}
\rho_{11}+\rho_{22} & \rho_{23} & -\rho_{13} \\
\rho_{32} & \rho_{11}+\rho_{33} & \rho_{12} \\
-\rho_{31} & \rho_{21} & \rho_{22}+\rho_{33}
\end{array}\right) \text {. }
$$

The map $\mathscr{M}: \rho_{I} \mapsto \rho_{1,2}$ is a completely positive tracepreserving (CPTP) map. It is obvious because the map $\rho_{I} \mapsto \rho_{O}$ is an isomorphism and expanding $\rho_{O}$ to $\rho_{O}^{\prime}$ is simply adding extra dimensions to Hilbert space which do not participate in constructing of the state $\rho_{O}^{\prime}$; that is, the spectrum of $\rho_{O}^{\prime}$ is equal to the spectrum of $\rho_{O}$ and hence of $\rho_{I}$. The partial trace is obviously a CPTP map, so the whole map $\mathscr{M}$ is CPTP. Despite such trivial construction of reduced states $\rho_{1,2}$ in general their spectrum is different from $\rho_{I}$ because their construction was out of quasifermionic antisymmetric subspace $\mathscr{H} \subset \mathrm{H}_{3} \otimes H_{3}$.

\section{Correlations between Different Degrees of Freedom of Qutrit}

The well-known Peres-Horodecki criterion or PPT criterion is a necessary condition for a mixed state to be separable. It states that if the density operator $\rho$ of a bipartite system is separable then partial transpose of one of the subsystems is positive. The partial transpose of the second subsystem of $\rho_{O}^{\prime}$ in matrix form is the following:

$$
\begin{aligned}
\rho_{O}^{\prime P T} & =\frac{1}{2} \\
& \left(\begin{array}{ccccccccc}
0 & 0 & 0 & 0 & -\rho_{11} & -\rho_{21} & 0 & -\rho_{12} & -\rho_{22} \\
0 & \rho_{11} & \rho_{21} & 0 & 0 & 0 & 0 & -\rho_{13} & -\rho_{23} \\
0 & \rho_{12} & \rho_{22} & 0 & \rho_{13} & \rho_{23} & 0 & 0 & 0 \\
0 & 0 & 0 & \rho_{11} & 0 & -\rho_{31} & \rho_{12} & 0 & -\rho_{32} \\
-\rho_{11} & 0 & \rho_{31} & 0 & 0 & 0 & \rho_{13} & 0 & -\rho_{33} \\
-\rho_{12} & 0 & \rho_{32} & -\rho_{13} & 0 & \rho_{33} & 0 & 0 & 0 \\
0 & 0 & 0 & \rho_{21} & \rho_{31} & 0 & \rho_{22} & \rho_{32} & 0 \\
-\rho_{21} & -\rho_{31} & 0 & 0 & 0 & 0 & \rho_{23} & \rho_{33} & 0 \\
-\rho_{22} & -\rho_{32} & 0 & -\rho_{23} & -\rho_{33} & 0 & 0 & 0 & 0
\end{array}\right) .
\end{aligned}
$$

It can be easily verified that $\rho_{O}^{\prime P T}$ has negative values for various $\rho_{I}$ states; that is, it is an entangled state. Actually, with the aid of entanglement monotone called negativity it is possible to show that this state is always entangled.

The entanglement monotone $E$ is nonnegative linear functional over state $\rho$ with two main properties:

(1) The monotone is a convex functional: $E\left(\sum_{i} p_{i} \rho_{i}\right) \leq$ $\sum_{i} p_{i} E\left(\rho_{i}\right)$.

(2) The functional is an entanglement monotone, that is, it does not increase on average under local quantum operations and classical communication (LOCC).

Most of the entanglement monotones, like distillability monotones and monotones of formation, are immensely difficult to compute. The more easily countable measure of entanglement is called negativity monotone. It is defined as follows:

$$
E(\rho)=\frac{\left\|\rho^{P T}\right\|_{1}-1}{2}=\sum_{i} \frac{\left|\lambda_{i}\right|-\lambda_{i}}{2}
$$

where $\|X\|_{1}=\operatorname{Tr}\left(\sqrt{X^{\dagger} X}\right)$ is a trace norm. In case of Hermitian operators it is just a sum of modules of negative eigenvalues.

Now it is possible to show that in case of initial pure states $\rho_{I}=|\psi\rangle\langle\psi|$ their negativity is $E=1 / 2$ and in case of maximally chaotic state $\rho_{I}=\left(\begin{array}{ccc}1 / 3 & 0 & 0 \\ 0 & 1 / 3 & 0 \\ 0 & 0 & 1 / 3\end{array}\right) E=1 / 3$. More formally, one can use another more convenient entanglement monotone for pure states $|\psi\rangle \in H_{A} \otimes H_{B}$ : the entropy of the subsystem $S=S_{A}=S_{B}=-\operatorname{Tr} \rho_{A} \ln \rho_{A}$. In our case if the initial state $\rho_{I}=|\psi\rangle\langle\psi|$ and $|\psi\rangle=a_{1}\left|e_{1}\right\rangle+a_{2}\left|e_{2}\right\rangle+a_{3}\left|e_{3}\right\rangle$ then the density matrices of quasifermion subspaces are as follows:

$$
\begin{aligned}
\rho_{1} & =\rho_{2} \\
& =\frac{1}{2}\left(\begin{array}{ccc}
\left|a_{1}\right|^{2}+\left|a_{2}\right|^{2} & a_{2} a_{3}^{*} & -a_{1} a_{3}^{*} \\
a_{2}^{*} a_{3} & \left|a_{1}\right|^{2}+\left|a_{3}\right|^{2} & a_{1} a_{2}^{*} \\
-a_{1}^{*} a_{3} & a_{1}^{*} a_{2} & \left|a_{2}\right|^{2}+\left|a_{3}\right|^{2}
\end{array}\right) .
\end{aligned}
$$


A simple technical calculation shows that nonzero eigenvalues of this matrix are $\lambda_{1,2}=1 / 2$. This means that the entanglement measure of $\rho_{O}^{\prime}$ is $S=\ln 2$ for all initial pure states $\rho_{I}$. Thus the negativities $E$ of all pure states $\rho_{O}^{\prime}$ are also equal to each other. En elementary calculation for an arbitrary pure state shows that $E=1 / 2$.

The previous considerations about pure states negativities prove that for two different mixed states with equal coefficients $\rho_{I 1}=p_{1}\left|\psi_{1}\right\rangle\left\langle\psi_{1}\left|+p_{2}\right| \psi_{2}\right\rangle\left\langle\psi_{2}\left|+p_{3}\right| \psi_{3}\right\rangle\left\langle\psi_{3}\right|$ and $\rho_{I 2}=$ $p_{1}\left|\phi_{1}\right\rangle\left\langle\phi_{1}\left|+p_{2}\right| \phi_{2}\right\rangle\left\langle\phi_{2}\left|+p_{3}\right| \phi_{3}\right\rangle\left\langle\phi_{3}\right|$ negativities are equal to each other. Thus to analyze all mixed states it is enough to consider a general density matrix:

$$
\rho_{I}=\left(\begin{array}{ccc}
p_{1} & 0 & 0 \\
0 & p_{2} & 0 \\
0 & 0 & p_{3}
\end{array}\right) .
$$

The partial transpose of $\rho_{O}^{\prime}$ then will be the following:

$$
=\frac{1}{2}\left(\begin{array}{ccccccccc}
0 & 0 & 0 & 0 & -p_{1} & 0 & 0 & 0 & -p_{2} \\
0 & p_{1} & 0 & 0 & 0 & 0 & 0 & 0 & 0 \\
0 & 0 & p_{2} & 0 & 0 & 0 & 0 & 0 & 0 \\
0 & 0 & 0 & p_{1} & 0 & 0 & 0 & 0 & 0 \\
-p_{1} & 0 & 0 & 0 & 0 & 0 & 0 & 0 & -p_{3} \\
0 & 0 & 0 & 0 & 0 & p_{3} & 0 & 0 & 0 \\
0 & 0 & 0 & 0 & 0 & 0 & p_{2} & 0 & 0 \\
0 & 0 & 0 & 0 & 0 & 0 & 0 & p_{3} & 0 \\
-p_{2} & 0 & 0 & 0 & -p_{3} & 0 & 0 & 0 & 0
\end{array}\right) .
$$

Its negative eigenvalues are the negative solutions of the thirdorder equation:

$$
\lambda^{3}-\left(p_{1}^{2}+p_{2}^{2}+p_{3}^{2}\right) \lambda+2 p_{1} p_{2} p_{3}=0 .
$$

Careful analysis shows that for every distribution $p_{i}$ there is only one negative solution. The infimum of negativity is situated at maximally chaotic state $p_{1}=p_{2}=p_{3}=1 / 3$ and is equal to $E=1 / 3$.

\section{Conclusions}

In this paper the simplest nontrivial case of quantum correlations inside qutrit was analyzed. It was demonstrated that for arbitrary mixed qutrit state the negativity is nonzero for corresponding virtual bipartite system of antisymmetrized qutrits. Thus the correlations between the degrees of freedom of qutrit emerge. These considerations could be generalized for qudits with more degrees of freedom in a straightforward manner.

\section{Competing Interests}

The authors declare that there are no competing interests regarding the publication of this paper.

\section{References}

[1] E. Schrödinger, "Die gegenwärtige Situation in der Quantenmechanik," Naturwissenschaften, vol. 23, no. 48, pp. 807-812, 1935.

[2] C. H. Bennett and S. J. Wiesner, "Communication via oneand two-particle operators on Einstein-Podolsky-Rosen states," Physical Review Letters, vol. 69, no. 20, pp. 2881-2884, 1992.

[3] C. H. Bennett, G. Brassard, C. Crépeau, R. Jozsa, A. Peres, and W. K. Wootters, "Teleporting an unknown quantum state via dual classical and Einstein-Podolsky-Rosen channels," Physical Review Letters, vol. 70, no. 13, pp. 1895-1899, 1993.

[4] A. Steane, "Quantum computing," Reports on Progress in Physics, vol. 61, no. 2, pp. 117-173, 1998.

[5] N. Linden, "Good dynamics versus bad kinematics: is entanglement needed for quantum computation?" Physical Review Letters, vol. 87, no. 4, Article ID 047901, 2001.

[6] P. W. Shor and J. Preskill, "Simple proof of security of the BB84 quantum key distribution protocol," Physical Review Letters, vol. 85, no. 2, pp. 441-444, 2000.

[7] H. Aschauer and H. J. Briegel, "Private entanglement over arbitrary distances, even using noisy apparatus," Physical Review Letters, vol. 88, no. 4, Article ID 047902, 2002.

[8] M. Kitagawa and M. Ueda, "Squeezed spin states," Physical Review A, vol. 47, no. 6, pp. 5138-5143, 1993.

[9] J. Hald, J. L. Sørensen, C. Schori, and E. S. Polzik, "Spin squeezed atoms: a macroscopic entangled ensemble created by light," Physical Review Letters, vol. 83, no. 7, pp. 1319-1322, 1999.

[10] C. H. Bennett, H. J. Bernstein, S. Popescu, and B. Schumacher, "Concentrating partial entanglement by local operations," Physical Review A, vol. 53, no. 4, pp. 2046-2052, 1996.

[11] C. H. Bennett, D. P. DiVincenzo, J. A. Smolin, and W. K. Wootters, "Mixed-state entanglement and quantum error correction," Physical Review A, vol. 54, no. 5, pp. 3824-3851, 1996.

[12] V. Vedral, M. B. Plenio, M. A. Rippin, and P. L. Knight, "Quantifying entanglement," Physical Review Letters, vol. 78, no. 12, pp. 2275-2279, 1997.

[13] W. K. Wootters, "Entanglement of formation of an arbitrary state of two qubits," Physical Review Letters, vol. 80, no. 10, pp. 2245-2248, 1998.

[14] A. Peres, "Separability criterion for density matrices," Physical Review Letters, vol. 77, no. 8, pp. 1413-1415, 1996.

[15] M. Horodecki and P. H. Horodecki, "Separability of mixed states: necessary and sufficient conditions," Physics Letters A, vol. 223, no. 1-2, pp. 1-8, 1996.

[16] K. Zyczkowski, P. Horodecki, A. Sanpera, and M. Lewenstein, "Volume of the set of separable states," Physical Review. A, vol. 58, no. 2, pp. 883-892, 1998.

[17] J. Eisert and M. B. Plenio, "A comparison of entanglement measures," Journal of Modern Optics, vol. 46, no. 1, pp. 145-154, 1999.

[18] G. Vidal and R. F. Werner, "Computable measure of entanglement," Physical Review A, vol. 65, no. 3, Article ID 032314, 2002.

[19] M. A. Man'ko and V. I. Man'ko, "Entanglement and other quantum correlations of a single qudit state," International Journal of Quantum Information, vol. 12, no. 7-8, Article ID 1560006, 2014.

[20] M. A. Man'ko and V. I. Man'ko, "Properties of nonnegative Hermitian matrices and new entropic inequalities for noncomposite quantum systems," Entropy, vol. 17, no. 5, pp. 2876-2894, 2015. 
[21] A. R. Kessel and N. M. Yakovleva, "Implementation schemes in NMR of quantum processors and the Deutsch-Jozsa algorithm by using virtual spin representation," Physical Review A, vol. 66, no. 6, Article ID 062322, 2002.

[22] E. O. Kiktenko, A. K. Fedorov, and V. I. Man'ko, “Teleportation in an indivisible quantum system," Quantum Measurements and Quantum Metrology, vol. 3, no. 1, pp. 15-19, 2016.

[23] V. N. Chernega, O. V. Man’ko, and V. I. Man’ko, "Generalized qubit portrait of the qutrit-state density matrix," Journal of Russian Laser Research, vol. 34, no. 4, pp. 383-387, 2013.

[24] A. K. Fedorov, E. O. Kiktenko, O. V. Man’ko, and V. I. Man’ko, "Multilevel superconducting circuits as two-qubit systems: operations, state preparation, and entropic inequalities," Physical Review A, vol. 91, no. 4, Article ID 042312, 2015.

[25] E. O. Kiktenko, A. K. Fedorov, A. A. Strakhov, and V. I. Man'ko, "Single qudit realization of the Deutsch algorithm using superconducting many-level quantum circuits," Physics Letters A, vol. 379, no. 22-23, pp. 1409-1413, 2015.

[26] J. Schliemann, J. I. Cirac, M. Kuś, M. Lewenstein, and D. Loss, "Quantum correlations in two-fermion systems," Physical Review A, vol. 64, no. 2, 2001.

[27] P. Zanardi, "Quantum entanglement in fermionic lattices," Physical Review A, vol. 65, no. 4, Article ID 042101, 2002.

[28] K. Eckert, J. Schliemann, D. Bruß, and M. Lewenstein, "Quantum correlations in systems of indistinguishable particles," Annals of Physics, vol. 299, no. 1, pp. 88-127, 2002.

[29] F. Benatti, R. Floreanini, and K. Titimbo, "Entanglement of identical particles," Open Systems \& Information Dynamics, vol. 21, no. 1-2, Article ID 1440003, 2014.

[30] A. Reusch, J. Sperling, and W. Vogel, "Entanglement witnesses for indistinguishable particles," Physical Review A, vol. 91, no. 4, Article ID 042324, 2015. 


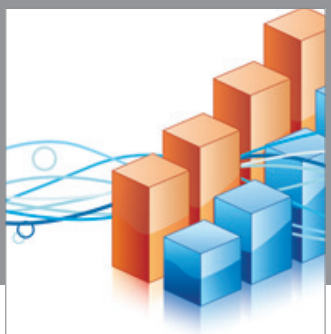

Advances in

Operations Research

vatem alat4

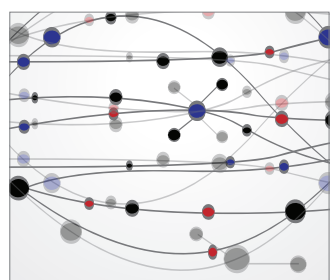

\section{The Scientific} World Journal
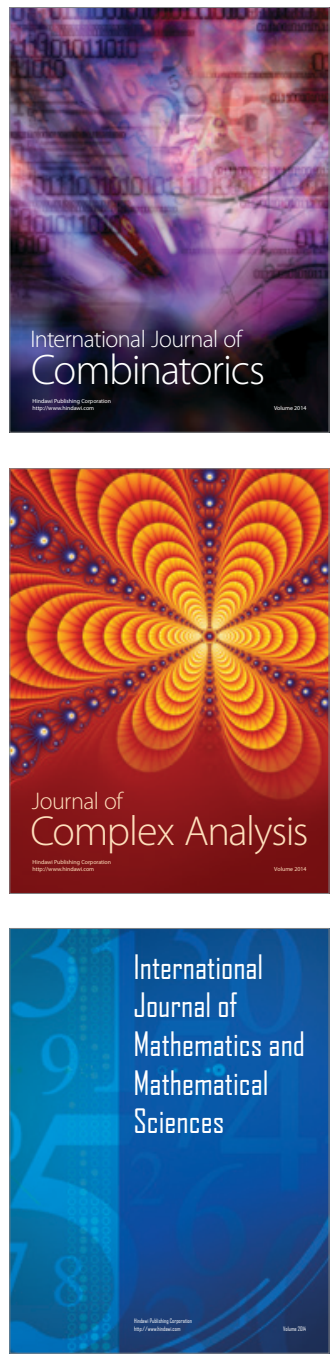
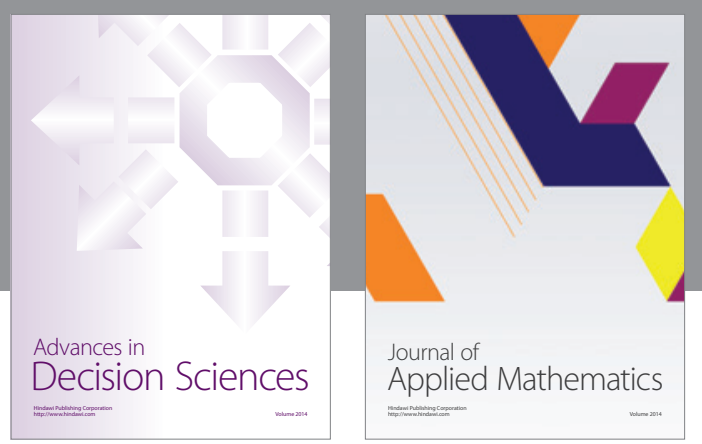

Algebra

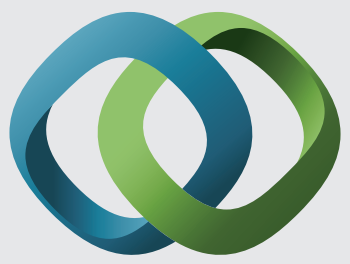

\section{Hindawi}

Submit your manuscripts at

http://www.hindawi.com
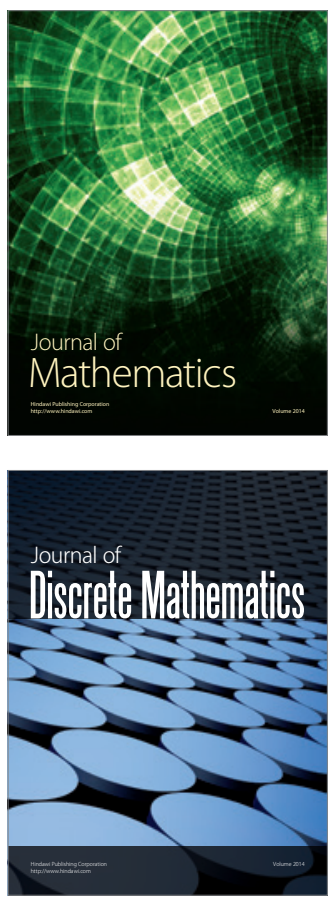

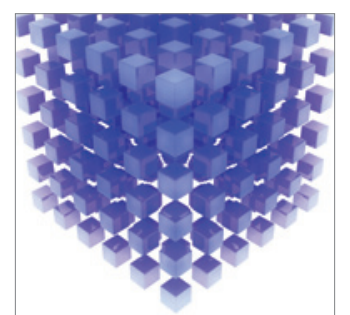

Mathematical Problems in Engineering
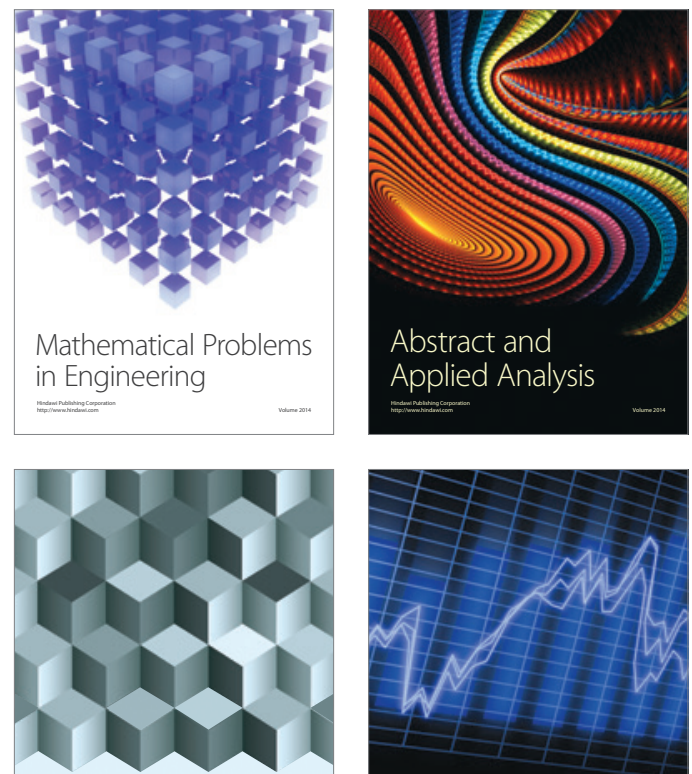

Journal of

Function Spaces

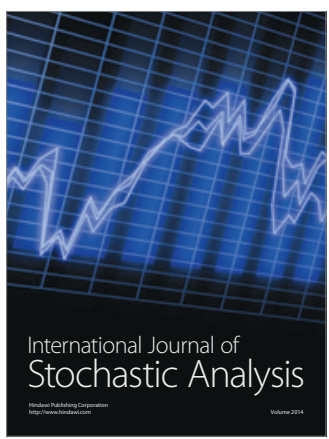

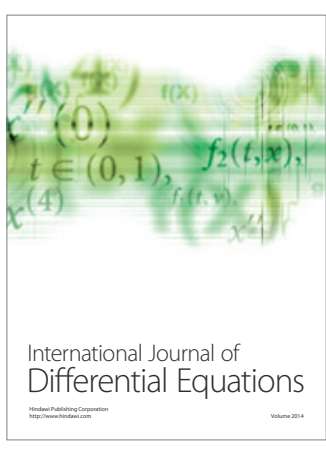
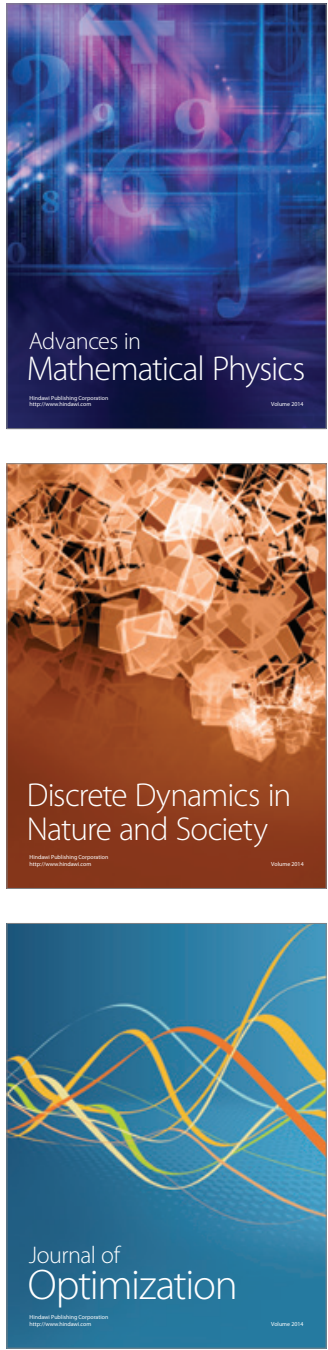